\title{
POLARIZED INFRARED EMISSION FROM DUST
}

\author{
ROGER H. HILDEBRAND \\ The University of Chicago
}

At the beginning of this decade what we knew about polarization of far-infrared emission from dense clouds was that some very good observers had looked for it and had not found it. Gull et al. (1978) had shown that the degree of polarization in Orion was not more than $2 \%$. That information provided an important guide but very little encouragement for later efforts. There was reason to doubt whether the mechanisms invoked to explain the alignment of dust grains in the diffuse intercloud medium would operate in dense clouds; whether the strengths of the magnetic fields in dense clouds would be sufficiently greater than those in the intercloud medium to overcome the higher rate at which gas collisions would destroy alignment; and whether the field, if sufficient locally, would have enough large-scale order to give measurable polarization in far-infrared observations with large beams and large column depths.

We have since found that the thermal emission from dense clouds is in fact polarized to an extent consistent with the earlier limits: the effect exists.

It is evident from the examples shown in Table 1 and Figures 1-3 that the aligning field has large-scale order, not only in the dust columns falling within single beams, but also over regions several beams in extent. At the center of the galaxy, in the dust ring at Sgr A, the field direction is nearly uniform over a region some 8 pc from north to south (Fig. 1), and there is similar uniformity on a much finer scale in the $12.5 \mu \mathrm{m}$ emission from the "Northern Arm" near the center of that region (Smith, Roche, and Aitken, 1988; Fig. 2).

We still do not know the strength of the magnetic field required for aligning grains in either tenuous or dense clouds, but Zeeman measurements have shown how the field strength, $B$, varies with the gas density, $n$ (see reviews by Mouschovias (1987) and by Troland and Heiles (1986)). The results are consistent with an increase in $B$ in proportion to $\sqrt{n}$ but not until $n \geq 100 / \mathrm{cm}^{3}$, that is; $B<B_{0} \sqrt{\left(n / n_{0}\right)}$, where $B_{0}$ and $n_{0}$ are the intercloud values ( $\mathrm{Sgr} A$ may be an exception). Since the torques that align grains are proportional to $B^{2}$, and the rate of gas damping is proportional to $n$, the ratio of the time available to the time required for alignment should be proportional to $n / B^{2}$. We may therefore conclude that any magnetic alignment mechanism that is inadequate or marginal in the intercloud medium will fail altogether in a dense cloud unless the effect of gas damping can be suppressed (as in suprathermal rotation).

In the tenuous expanses of the Galaxy, the evidence for magnetic alignment and, in particular, paramagnetic alignment of spinning grains, is compelling. Both 


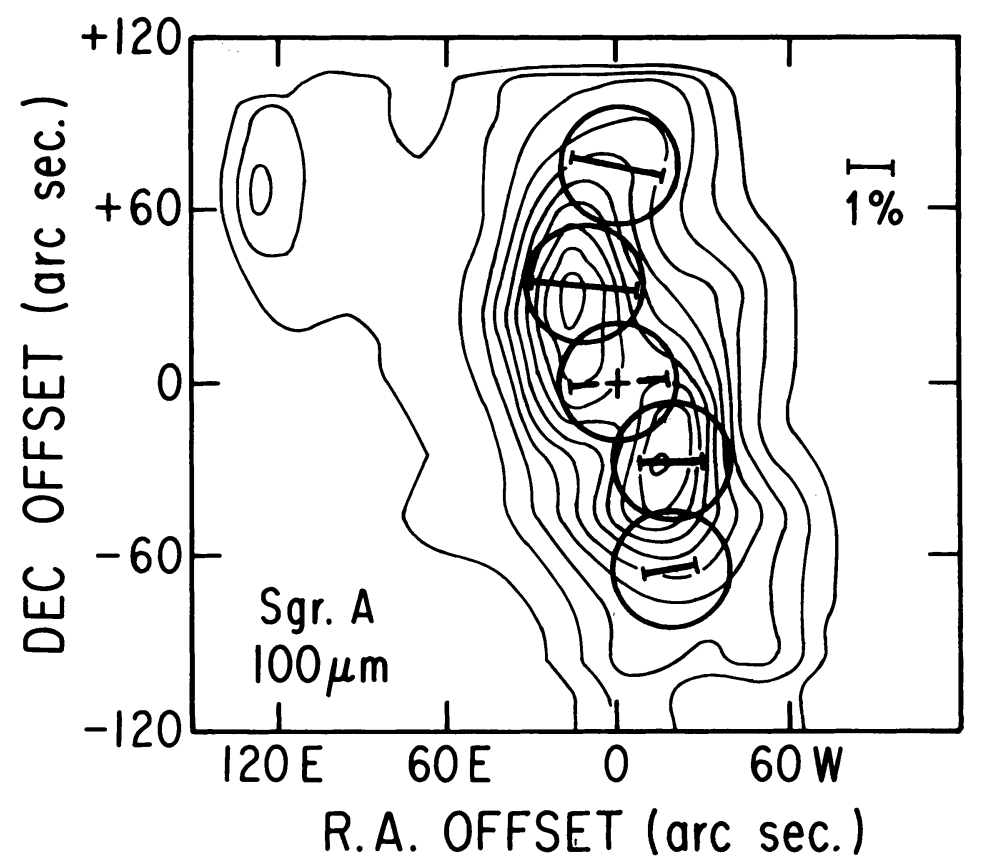

Fig. 1. Polarization of the $100 \mu \mathrm{m}$ emission from the dust ring surrounding Sgr A (Hildebrand et al., 1988). The map of flux densities is by Davidson, Harvey, Lester, Morris, and Werner (1988). The circles show the positions of the beams used in the polarimetry: the enclosed bars show the degree and direction of the polarization ( $E$-vector) as measured with the University of Chicago far-infrared polarimeter on the Kuiper Airborne Observatory. Offsets are measured from $17^{\mathrm{h}} 42^{\mathrm{m}} 29.4^{\mathrm{s}},-28^{\circ} 59^{\prime} 19^{\prime \prime}(1950)$.

the strength and the configuration of the field as derived from the polarization of starlight are confirmed in detail by investigations of synchrotron radiation, Zeeman splitting, and Faraday rotation. Nevertheless, one may consider whether other alignment processes could account for the polarized emission from dense clouds.

I have reviewed elsewhere (Hildebrand, 1987, 1988) the various mechanisms that have been considered to account for grain alignment: the streaming of gas or photons through dust clouds, ferromagnetic alignment (as displayed by compass needles), the Roland effect of spinning grains with surface charges, and paramagnetic relaxation (Davis-Greenstein, 1951) as enhanced by suprathermal rotation or superparamagnetism. I will discuss here only what the polarization of thermal emission tells us about these mechanisms.

What we can say about the mechanism now that we could not say in 1980 is that some of the conditions we thought might be necessary for alignment in dense clouds apparently don't matter. Alignment by bulk streaming of gas with respect 


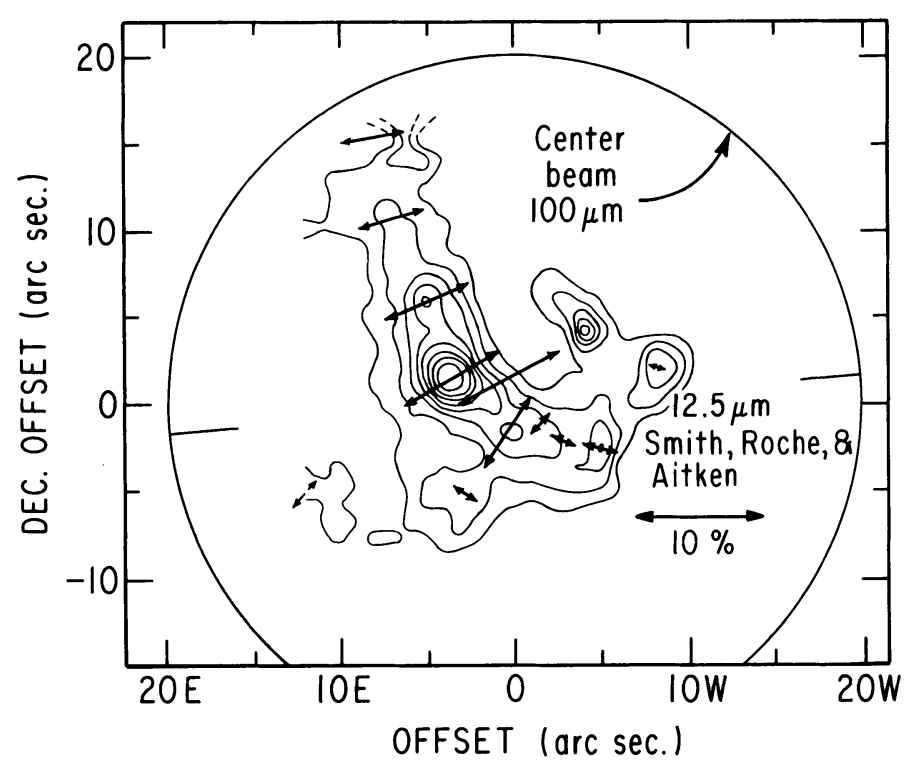

Fig. 2. Comparison of $12.5 \mu \mathrm{m}$ and $100 \mu \mathrm{m}$ polarimetry of Sgr A. The contours show the flux density at 8-13 $\mu \mathrm{m}$ and the arrows the polarization at $12.5 \mu \mathrm{m}$ (Smith, Roche, and Aitken, 1988). The large circle and radial tick marks show the central beam (offset 0,0 of Fig. 1) and position angle for observations at $100 \mu \mathrm{m}$ (Hildebrand et al., 1988).

to dust (Gold, 1952) very likely does occur somewhere in the universe, but it cannot account for the polarization found in any of the measurements shown here. In the case of Orion, for example (Fig. 3), there is indeed high velocity gas streaming from the vicinity of the Becklin-Neugebauer object (BN), but it is in the wrong direction to account for the observed polarization. Streaming would tend to put the long axis of the grains, and hence the $E$-vector of the emitted polarization, along the direction of the flow, in this case at a position angle of about $125^{\circ}$. What we find is a position angle of $22^{\circ}$. We also find that the polarization is just as great and in nearly the same direction in a region 1.5 arc. min. south of $\mathrm{BN}$ where there is no high velocity flow in any direction.

Alignment by streaming of high energy photons can, in principle, produce alignment in the manner of streaming gas. At Sgr A (Fig. 1) there is a source of ultraviolet photons at the center and dust emission peaks to the north and south, but the flux of UV photons is too feeble to overcome the effects of gas collisions, and the photons, like the gas molecules in Orion, are moving in the wrong direction to explain the observed polarization.

Alignment by absorption of low energy photons $(\lambda \ll 2 \pi a)$ for which the intrinsic angular momentum exceeds the product of the linear momentum and grain radius can, in principle, cause the grains to spin with their long axes perpendicular to 
TABLE 1

POLARIZATION IN ORION AND THE Galactic CENTER

\begin{tabular}{|c|c|c|c|c|c|}
\hline Object & $\begin{array}{l}\text { Offset }^{a} \\
\operatorname{arc~sec}\end{array}$ & $\begin{array}{l}\text { Beam diam. } \\
\text { arc sec }\end{array}$ & $\begin{array}{c}\lambda \\
\mu \mathrm{m}\end{array}$ & $\begin{array}{l}\mathrm{P} \\
\%\end{array}$ & $\Phi^{\mathrm{b}}$ \\
\hline \multirow[t]{4}{*}{ Orion $^{c}$} & 0,0 & 120 & 77 & $2.2 \pm 0.4$ & $16 \pm 8$ \\
\hline & 0,0 & 60 & 100 & $1.67 \pm 0.2$ & $22 \pm 4$ \\
\hline & 0,0 & 60 & 270 & $1.67 \pm 0.2$ & $24 \pm 6$ \\
\hline & $12 \mathrm{~W}, 88 \mathrm{~S}$ & 60 & 270 & $1.65 \pm 0.3$ & $28 \pm 7$ \\
\hline \multirow[t]{5}{*}{ Sgr A } & $0,75 \mathrm{~N}$ & 40 & 100 & $2.3 \pm 0.4$ & $77 \pm 5$ \\
\hline & $12 \mathrm{E}, 34 \mathrm{~N}$ & 40 & 100 & $2.8 \pm 0.3$ & $85 \pm 3$ \\
\hline & 0,0 & 40 & 100 & $2.4 \pm 0.3$ & $95 \pm 3$ \\
\hline & $18 \mathrm{~W}, 28 \mathrm{~S}$ & 40 & 100 & $1.5 \pm 0.3$ & $93 \pm 5$ \\
\hline & $18 \mathrm{~W}, 65 \mathrm{~S}$ & 40 & 100 & $1.2 \pm 0.4$ & $100 \pm 8$ \\
\hline \multicolumn{6}{|c|}{ Offiets from $5^{\mathrm{h}} 32^{\mathrm{m}} 46.8^{8},-5^{\circ} 24^{\prime} 19^{\prime \prime}:$ Orion, BN } \\
\hline \multicolumn{6}{|c|}{$\begin{array}{l}\text { b Position angle of the E-vector in degrees east from north. } \\
\text { ' } R \text { Results at } 77 \mu \mathrm{m} \text { are from University College London (Cudlip et al., 1982); all other } \\
\text { from the University of Chicago (Hildebrand, Dragovan, and Novak 1984; Dragovan, } 1986 \\
\text { Hildebrand et al., 1988). }\end{array}$} \\
\hline
\end{tabular}

the direction of the incident flux (Harwit, 1970). For that mechanism to cause alignment in the regions we have examined, it would be necessary to reduce the gas density by many orders of magnitude, and since the strongest sources of low energy photons are the infrared intensity peaks, we should not see any polarization when we look directly at them, but we do.

The only alignment mechanism which fits the facts about polarization of visible starlight and about polarization of far-infrared emission is the Davis-Greenstein mechanism (1951), the removal by paramagnetic relaxation of rotation perpendicular to the field, $B$, so that the grains spin only about $B$. Work against inelastic forces and the Barnett effect (flipping of electron spins) insure that the short axis of a grain, the axis of maximum rotational inertia, will be aligned with the spin axis and hence with $B$ (Purcell, 1979).

The difficulty with the Davis-Greenstein mechanism has been that the rate of alignment, calculated for grains made of ordinary paramagnetic materials under the conditions in the arms of the Galaxy, is slower by about one order of magnitude than the calculated rate at which gas collisions should destroy the alignment. The situation should be worse in a dense cloud. Purcell (1979) has proposed that this difficulty could be overcome by suprathermal rotation; that is by rotation of the grains at kinetic energies much greater than $k T$. He argues that special sites on the surface of a grain may have characteristics that could favor emission of photoelectrons, fusion of hydrogen atoms, or inelastic collisions with gas molecules, i.e. processes that deliver impacts to the grain. If these sites are unevenly distributed over the surface, the impacts will drive the grain repeatedly in the same direction. The rotational kinetic energy could then become thousands of times greater than 


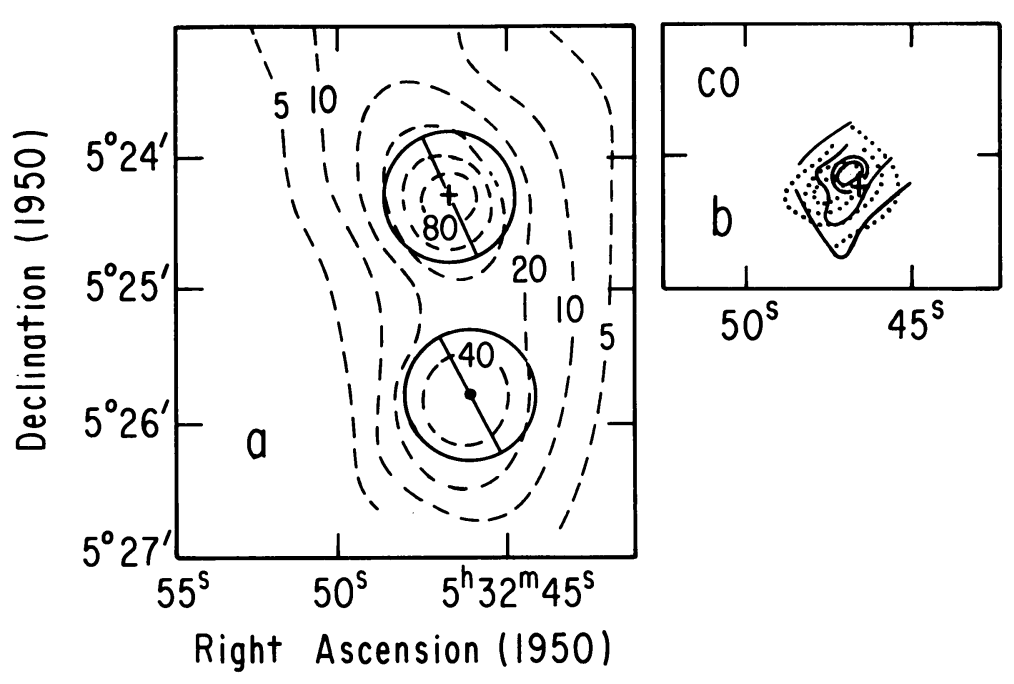

Fig. 3. (a) Polarization of the $270 \mu \mathrm{m}$ emission from Orion (Hildebrand, Dragovan, and Novak, 1982; Dragovan, 1986). The circles show the beam positions superimposed on the $400 \mu \mathrm{m}$ map of Keene, Hildebrand, and Whitcomb (1982). The cross is at BN. The contours are labeled in percent of the peak flux. The lines through the circles show the position angles of the polarization ( $E$-vector) as measured with the University of Chicago far-infrared polarimeter on the Kuiper Airborne Observatory. (b) Red-shifted and blue-shifted $C O \mathrm{~J}=3-2$ emission in the vicinity of $\mathrm{BN}$ (cross) as measured by Erickson et al., 1982 . (Solid curves for -59 to $+2 \mathrm{~km} / \mathrm{s}$, dotted curves for +16 to +75 $\mathrm{km} / \mathrm{s}$.) The contours are at $0.9,0.8,0.6$, and 0.4 of the peak values of integrated emission.

$k T$ where $T$ is the temperature of the grain or the gas. The consequence of such suprathermal rotation would not be to reduce the time for alignment - the angular momentum to be removed would increase in proportion to the angular velocity but rather that the time available for alignment would not be limited by gas damping but would continue for as long as the grain was driven about a particular axis. What is necessary, then, is to have a supply of high energy photons, or hydrogen atoms, or a large difference between the temperatures of gas and dust, and for the special sites to persist long enough for the grains to become aligned. If the accretion of a monolayer destroys the special sites, then there is not enough time to align grains with ordinary magnetic susceptibilities.

Our results show that suprathermal rotation is either extremely efficient or unnecessary. In Sgr A (Figs. 1 and 2) the degree of polarization is not correlated with proximity to the strong central source of UV photons. In Orion (Fig. 3) the polarization in the cool submillimeter peak south of BN is just as great as at BN itself. At the submillimeter peak one expects mostly infrared photons, molecular hydrogen, cool gas and dust, and grains very likely covered with thick mantles. These are not the conditions that should favor suprathermal rotation.

Spitzer and Tukey (1951) have argued that grains could become superparam- 
agnetic if violent collisions or other processes should cause iron atoms, or other ferromagnetic materials, to gather in clumps below the size that would permit interacting domains. The time required for alignment would then be much less than the gas damping time or the time for accretion of a monolayer. The alignment would still be inefficient for grains near equilibrium with the gas but would be very efficient for grains with suprathermal rotation.

Although a combination of superparamagnetism and suprathermal rotation would be more than sufficient to align grains in either tenuous or dense clouds, one cannot be altogether content with an explanation that rests on the failure of other mechanisms and that must work efficiently even where the conditions appear to be unfavorable. It will take more convincing evidence to settle the question.

Whatever the alignment mechanism may be, it causes comparable polarization in regions of vastly differing temperature, density, turbulence, magnetic field strength, and exposure to radiation. It may therefore be reasonable to assume that where polarization is observed, the alignment is saturated.

Our ignorance of grain properties and alignment mechanisms is such that we cannot use the degree of polarization at a particular point to derive the strength of the aligning field. But as shown by Davis (1951) and by Chandrasekhar and Fermi (1953), it is possible without reference to grain models or alignment processes to derive the field from a map of polarization vectors. In a dense cloud with known densities and known turbulent velocities, one can do with polarization of near- and far-infrared emission what those authors have done with the polarization of visible starlight. In Sgr A we estimate a field of at least 10 milligauss throughout the 3 pc dust ring. That may be the largest field in the Galaxy to fill a region of such an extent. Such a field must strongly influence the dynamics of the ring.

It is evident that observations of polarized infrared emission can now be broadly used to find the configuration and magnitude of magnetic fields in dense clouds whether or not we are able to resolve the questions that still haunt our theories of grain alignment.

ACKNOWLEDGEMENTS. I wish to acknowledge the participation of my students and former students, J. A. Davidson (now at Ames), M. Dragovan (now at Princeton), D. Gonatas, G. Novak (now at U Mass), S. R. Platt, and X. Wu, and collaborators, M. Morris and M. Werner in the far-infrared measurements presented here. I also wish to thank D. Aitken, B. Draine, and J. Mathis for many discussions and communications. This work was supported by NASA Grant No. NSG 2057 and NSF Grant No. AST 8513974.

\section{REFERENCES}

Aitken, D. K., Roche, P. F., Bailey, J. A., Briggs, G. P., Hough, J. H., and Thomas, J. A. 1986, $M$. N.R. A. S., 218,363 .

Chandrasekhar, S., and Fermi, E. 1953, Ap. J., 118, 113.

Cudlip, W., Furniss, I., King, K. J., and Jennings, R. E. 1982, M. N. R. A. S., 200, 1169.

Davidson, J. A., Harvey, P. M., Lester, D. F., Morris, M., and Werner, M. W. 1988, in preparation.

Davis, L., Jr., 1951, Phys. Rev., 81, 890. 
Davis, L., Jr., and Greenstein, J. L. 1951, Ap. J., 114, 206.

Dragovan, M. 1986, Ap. J., 308, 270.

Erickson, N. R., Goldsmith, P. F., Snell, R. L., Berson, R. L., Huguenin, G. R., Ulrich, B. L., and Lada, C. J. 1982, Ap. J., $261, \mathrm{~L} 103$.

Gold, T. $1952, M . N$. R. A. S., 112, 215.

Gull, G. E., Russell, R. W., Melnick, G., and Harwit, M. 1980, Ap. J., 85, 1379.

Harwit, M. 1970, Nature, 226, 61.

Hildebrand, R. H., Dragovan, M., and Novak, G. 1984, Ap. J., 284, L51.

Hildebrand, R. H. 1987, Astro. Lett. and Communications, 26, 263.

Hildebrand, R. H. 1988, Quart. J. R. A. S., in press.

Hildebrand, R. H., Davidson, J. A., Gonatas, D., Novak, G., Platt, S. R., and Wu, X. 1988. The Sgr A results presented here will be incorporated in a later publication (see also Werner et al., 1988).

Keene, J., Hildebrand, R. H., and Whitcomb, S. E. 1982, Ap. J., 252, L11.

Mouschovias, T. Ch. 1987, in Physical Processes in Interstellar Clouds, eds. G. E. Morfill and M. D. Scholer (Boston: Reidel), p. 455.

Novak, G. 1988, Ph.D. thesis, University of Chicago.

Novak, G:, Dragovan, M., Gonatas, D., Hildebrand, R. H., and Platt, S. R. 1988. (Submitted to Ap. J.).

Purcell, E. M. 1979, Ap. J., $231,404$.

Smith, C. H., Roche, P. F., and Aitken, D. K. 1988, in preparation. (See also Aitken et al., 1986.)

Spitzer, L. (Jr.), and Tukey, J. W. 1951, Ap. J., 114, 187.

Troland, T. H., and Heilas, C. 1986, Ap. J., \$01, 339.

Werner, M. W., Davidson, J. A., Hildebrand, R. H., Morris, M. R., Novak, G., and Platt, S. R. $1988, A p$. J., in press. 